\title{
Erratum
}

\section{Invariant measures and disintegrations with applications to Palm and related kernels}

\author{
Olav Kallenberg
}

Published online: 8 May 2007

(C) Springer-Verlag 2007

\section{Erratum to: Probab. Theory Relat. Fields DOI 10.1007/s00440-006-0053-y}

I am grateful to Mr. Daniel Gentner of Karlsruhe for calling my attention to some serious gaps in the proofs of two main results. In Theorem 2.4, the given proof applies only when $v g<\infty$. For a general $\nu$, choose $0<\tilde{g} \leq g$ with $\nu \tilde{g}<\infty$, and note that the associated $\varphi$-kernel equals $\tilde{\varphi}_{s}=r(s) \varphi_{s}$, where $r(s)=1 / \varphi_{s} \tilde{g}$. Hence,

$$
v=\int \tilde{g}(s) v(d s) \tilde{\varphi}_{s}=\int \tilde{g}(s) r(s) v(d s) \varphi_{s}=\int m \mu(d m),
$$

where $\mu=(\tilde{g} r \cdot v) \circ \varphi^{-1}$. To get $\mu=(g \cdot v) \circ \varphi^{-1}$, we need to show that $(\tilde{g} r \cdot v) A=$ $(g \cdot v) A$ when $A=\varphi^{-1} M$ for a measurable $M \subset \mathcal{M}_{S}$. By (1) it is then enough to take $v=\varphi_{s}$, in which case both sides reduce to $1_{A}(s)$, by the $G$-invariance of $r$ and $A$.

In the proof of Theorem 3.5, we need to justify (8), which is far from obvious. Then fix any $r \in G$. For measurable functions $g \geq 0$ on $S \times T$, the invariance of $M$ and $v$ yields $\mu_{s} g_{s}=\left(\mu_{r s} \circ \theta_{r}\right) g_{s}, s \in S$ a.e. $v$, where $g_{s}(t)=g(s, t)$. Replacing $g$ by $g f$ for measurable $f \geq 0$ on $T$ gives $\left(g_{s} \cdot \mu_{s}\right) f=\left(g_{s} \cdot\left(\mu_{r s} \circ \theta_{r}\right)\right) f$ a.e. $v$. Since $M$ is $\sigma$-finite, we may choose $g>0$ with $M g<\infty$, so that $\mu_{s} g_{s}<\infty$ a.e. $v$. Since $T$ is Borel, we obtain $g_{s} \cdot \mu_{s}=g_{s} \cdot\left(\mu_{r s} \circ \theta_{r}\right)$ a.e. $v$, which implies $\mu_{s}=\mu_{r s} \circ \theta_{r}$ a.e. $v$.

Thanks again to Daniel for some helpful correspondence.

The online version of the original article can be found at http://dx.doi.org/10.1007/s00440-006-0053-y.

O. Kallenberg $(\varangle)$

Department of Mathematics and Statistics, Auburn University,

221 Parker Hall, Auburn, AL 36849, USA

e-mail:kalleoh@auburn.edu 